\title{
Logistics and epidemiology of endovascular treatment in acute ischemic stroke patients in the Netherlands
}

\author{
Maas W.J. ${ }^{1,2}$, Luijckx G.J. ${ }^{1}$, Uyttenboogaart M. ${ }^{1}$, Van der Zee D.J. ${ }^{3}$, Lahr M.M.H. ${ }^{2}$, Buskens E. ${ }^{2,3}$.
}

'University of Groningen, University Medical Center Groningen, Department of Neurology, Groningen, Netherlands.

2University of Groningen, University Medical Center Groningen, Health Technology Assessment, Department of Epidemiology, Groningen, Netherlands.

3University of Groningen, Department of Operations, Faculty of Economics \& Business, Groningen, Netherlands.

RATIONALE

The organizational model for acute stroke care delivery is currently receiving a great deal of attention.

The emergence of endovascular treatment (EVT) for patients facing large-vessel occlusions has opened up a new dimension in terms of acute stroke pathway setup and patient logistics.

Within the current "drip-and-ship" treatment paradigm, eligible patients may initially be admitted to primary stroke centers before being transferred to comprehensive stroke centers with EVT capacity (Figure 2).

Parameters that determine timely treatment with EVT within the organizational models include:
- Distances to primary and comprehensive stroke centers.
- Inter-hospital transfer of EVT candidates.
- Availability of trained physicians, interventionalists and other hospital staff.

Further dissemination of EVT could increase treatment rates, yet, could affect efficient use of scarce resources, effectiveness and quality of care.

Objective: To study the organizational model and logistics of EVT for ischemic stroke patients in the Netherlands.

\section{DESIGN}

- Simulation modeling approach for quantifying procedures of hospitals offering EVT in the Netherlands.

- Patient level data concerning pathway logistics both pre- and intra-hospital will be collected and used to build and validate the simulation models.

Results from previous studies will be expanded with results and data of trials performed in the 'Collaboration for new treatments of acute stroke' (CONTRAST) consortium to represent current patient logistics, time delays and outcomes.

Simulation experiments will be performed to assess effectiveness and efficiency of alternative network typologies, i.e. primary and comprehensive stroke services ("Drip and ship" approach) versus a service delivery based on comprehensive and centralized care ("Mothership" approach).

- The model will be expanded with cost-data to prepare for elaborate budget impact and cost-effectiveness analyses.

\section{OUTCOME MEASURES}

Primary outcomes include EVT treatment rates and patient disability according to the Modified Rankin Scale.

As secondary outcomes the onset-to-treatment time and resource use will be collected.

\section{PATHWAY DESCRIPTIONS}

Figure 1. Direct transport to comprehensive stroke centers with EVT capabilities.

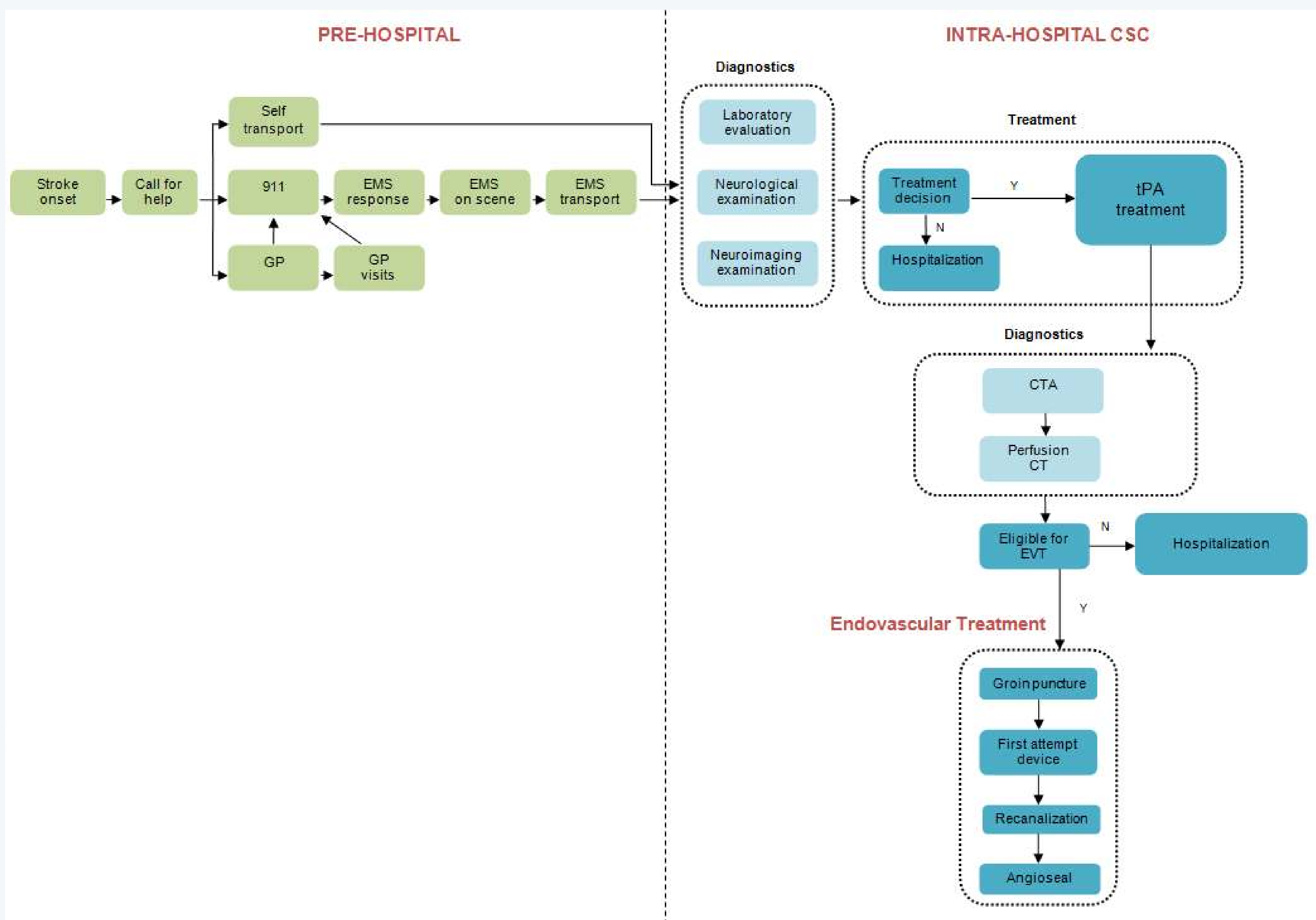

Figure 2. Indirect transport of EVT candidates via primary stroke centers.

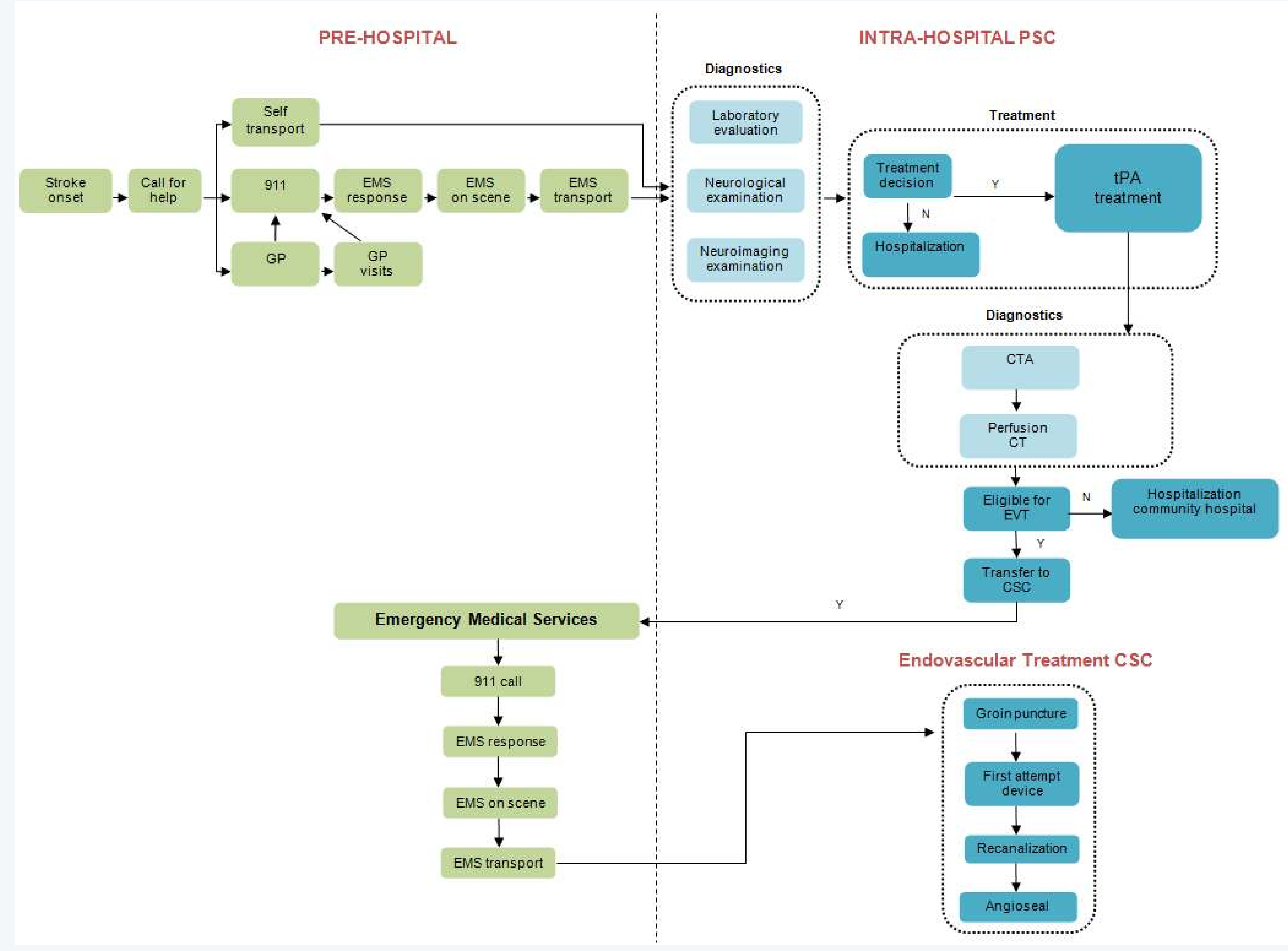

GP indicates general practitioner; EMS, emergency medical services; tPA, tissue plasminogen activator; $\mathrm{Y}=$ yes; $\mathrm{N}=$ no; CTA, computed tomography angiography; Perfusion $\mathrm{CT}$, perfusion computed tomography; $\mathrm{EVT}$, endovascular treatment; CSC, comprehensive stroke center; PSC, primary stroke center 